\title{
COMMISSION 29: STELLAR SPECTRA (SPECTRES STELLAIRES)
}

\author{
Report of Meetings, 22, 23, 25 and 28 August 1973
}

President: Y Fujita.

\section{First Session, 22 August 1973}

The Draft Report was accepted. The proposed members for the Organizing Committee were approved. New members and resignations of members were also approved.

The President reported about the activity of members who communicated with him since Circular No. 5 had been sent to each member on June 1, 1973.

The President asked the Chairmen of the Working Groups in attendance to report. D. C. Morton suggested L. N. Houziaux as his successor in the Working Group on 'Ultraviolet Stellar Spectra and related Ground-based Observations' $R$. Herman is willing to continue as Chairman of the Working Group on 'Be Stars' G. Cayrel de Strobel reported on the Working Group on 'Line Intensity Standards' as follows: The intercomparison of photometric calibration of stellar spectra, using Kienle's step filters has come to an end with negative results, e.g., calibration errors are not the major cause of divergence between equivalent widths measured with different equipments. Discrepancies of the order of $15 \%$ to $30 \%$ are still common, and as yet no clue exists as to what values are the best ones, an entirely new coordinated program should be undertaken to give a sort of metrologic tool to spectroscopists. As proposed earlier by Griffin, some spectra obtained in a double pass photoelectric spectrometer should be provided. The light of the source should obviously become available, both for northern and southern observatories. The best proposal could well be the integrated solar spectrum, which is also an invaluable comparison standard for stellar abundances. The solar spectrum can be obtained in stellar observatories either from diffusers of blue sky, the Moon, or, from stellar like sources as the minor planets supplying a large range of exposure times for comparison.

Copies of the memorandum to the members of Commissions $27,29,33,37$, and 42 , issued from the Moscow Bureau of Variable Stars in June, 1973, were distributed to members and the President invited the members to discuss it.

The proposal by M. Plavec for a symposium on Be and shell stars possibly in 1975 was supported. The proposal by L. H. Aller in connection with the 'Publication of Observational Data in Complex Stellar Spectra' was discussed and it was decided to re-discuss it in the Second Session on 28 August. Aller emphasized that the proper thing to do would be to publish the actual tracings. As spectrum synthesis methods become more and more popular, so becomes the necessity of presenting highresolution spectral intensity details and not just equivalent-widths.

Then the scientific session was continued with three invited speakers:

D. C. Morton: Ultraviolet Spectra of Bright O and B Stars by the OAO-3 Satellite.

S. B. Parsons: An Ultraviolet Stellar Spectroscopy Experiment on NASA Skylab.

J. Smoliński, J. L. Climenhaga, and K. O. Wright (read by Climenhaga): Blanketing Effect as related to Luminosity and Turbulence in Late-type Stars.

\section{Second Session, 28 August 1973}

The proposal by the President for the addition of a new member, Dr S. B. Parsons was accepted.

The proposal by Aller in connection with the 'Publication of Observational Data in Complex Stellar Spectra' was again discussed and the following two recommendations were accepted:

(1) Commission 29 recommends to astronomers who have data on the spectra of stars on mag- 
netic tape, that they fully document these data and make copies of the data available for other astronomers. Individual announcements about the availability of data should be made in each paper on stellar spectra.

(2) Commission 29 recommends to editors that they permit publishing of lists of line identifications and equivalent widths as well as profiles of lines in stellar spectra, for these data are fundamental to the study of stellar atmospheres. We urge the continued publication of atomic and molecular data on spectra of atoms, ions and molecules.

Then, the following statement was approved: Commission 29 fully endorses the proposal of the Commission 12 Working Group on the Solar Spectrum to prepare a fourth revision of the Rowland Table.

In discussing the future of the Commission the activity of each member was stressed, though new members are welcome.

The priorities for future research in the field of our Commission were shortly discussed.

Then followed a meeting of the Working Group on Be Stars, under the Chairmanship of Cayrel de Strobel. The following two papers were presented:

T. Kogure: Hydrogen Spectrum of Be Stars.

J. P. Maillard: Spectrum of Be Star $\gamma$ Cas for 1 to $2.5 \mu \mathrm{m}$.

\section{Joint Meetings}

Two Joint Meetings were organized by the President with the cooperation of A. H. Cook, President of Commission 14, and R. N. Thomas, President of Commission 36:

\section{JOINT MEETING ON 'LINE IDENTIFICATION IN STELLAR SPECTRA FOR} ATOMS AND MOLECULES'

Chairman: A. H. Cook.

(Commissions 14 and 29), August 23

C. M. Sitterly: Atomic Spectra-The Future Outlook.

J. E. Ross and L. H. Aller: Spectrum Synthesis and Spectral Line Identifications.

Chairman: L. H. Aller.

J. G. Phillips: Survey of Molecules in Astronomical Sources and of Recent Pertinent Laboratory Work.

Y. Fujita: Identification of Spectra in Violet and Infrared Regions of Carbon Stars.

\section{JOINT MEETING ON 'SPECTROSCOPY OF PECULIAR A-TYPE STARS'}

(Commissions 29 and 36), August 25

ChaIRMan: R. N. Thomas.

M. Hack: Surface Composition and Physical Structure of the Ap Stars.

D. S. Leckrone: The Ultraviolet Flux Envelopes of Ap Stars.

W. Buscombe: Variations in the Line Spectrum of A Supergiants.

Chatrman: M. Hack.

E. R. Mustel: Investigation of Ap Stars in U.S.S.R.

M. Smith: Abundance Studies of Am Stars across the HR Diagram.

Short comments by C. Meggesier and J. Dachs followed. 